\title{
Non-Euclidean Biosymmetries and Algebraic Harmony in Genomes of Higher and Lower Organisms
}

\author{
Sergey Petoukhov ${ }^{1,2 *}$, Elena Petukhova ${ }^{1}$, and Vitaly Svirin ${ }^{1,2}$ \\ ${ }^{1}$ Mechanical Engineering Research Institute of RAS, RU-101990, Moscow, Russia \\ ${ }^{2}$ Moscow State Conservatory, Russia, RU-125009, Moscow, Russia
}

\begin{abstract}
The article is devoted to the study of the relationship of non-Euclidean symmetries in inherited biostructures with algebraic features of information nucleotide sequences in DNA molecules in the genomes of eukaryotes and prokaryotes. These genomic sequences obey the universal hyperbolic rules of the oligomer cooperative organization, which are associated with the harmonic progression $1 / 1,1 / 2,1 / 3, . ., 1 / n$. The progression has long been known and studied in various branches of mathematics and physics. Now it has manifested itself in genetic informatics. The performed analysis of the harmonic progression revealed its connection with the cross-ratio, which is the main invariant of projective geometry. This connection consists in the fact that the magnitude of the cross-ratio is the same and is equal to $4 / 3$ for any four adjacent members of this progression. The long DNA nucleotide sequences have fractal-like structure with so called epi-chains, whose structures are also related to the harmonic progression and the projective-geometrical symmetries. The received results are related additionally to a consideration of DNA double helix as helical antenna. This fact of the connection of genetic informatics with the main invariant of projective geometry can be used to explain the implementation of some non-Euclidean symmetries in genetically inherited structures of living bodies.
\end{abstract}

\section{Introduction}

The phenomena of the symmetric inherited structure of biological bodies have been the subject of research since ancient times. The importance of studying these phenomena is described, for example, in classic books $[1,2]$. At the same time, until recently, all attention was paid to the biosymmetries of similarity, which are described using a group of geometric similarity transformations. According to F. Klein's Erlangen program, this group of transformations underlies Euclidean geometry and includes transformations of translations, rotations, specular reflections and scaling. At the same time, the hypothesis by V.I. Vernadsky on the possible important morphological significance of non-Euclidean symmetries in living bodies is widely known.

Clarification of the geometric foundations of morphology entails shifts in various areas of biomechanics and physiology: biomechanics of postures, biomechanics of growth and motor movements, psychophysics, etc. In the variety of organic forms, multiblock structures are of particular interest, the components of which are mutually conjugated on the basis of certain algorithms, repeated on the most diverse lines and branches of biological evolution. The general rule of such varieties, consisting of blocks or motivic units, is that the previous motivic unit is translated into the subsequent one by some fixed Euclidean or non-
Euclidean transformation. In other words, so organized segmented varieties have a cyclic group of automorphisms, and their motivic units are arranged along the orbit of the corresponding cyclic group. Earlier, in the works of the Institute of Mechanical Engineering Research Institute of the Russian Academy of Sciences, a wide representation in the inherited configurations of the set of living bodies of nonEuclidean symmetries was revealed, which are based on the groups of projective and Möbius transformations, which generalize the group of similarity transformations and contain it as their subgroup [3].

Genetically inherited morphological structures must be structurally linked to the molecular genetic system for their genetically coding and transmission along the chain of generations. Therefore, it is important to look for traces of symmetries and invariants of non-Euclidean transformations in the genetic informatics of living bodies, including in the information texts of DNA heredity molecules carrying genetic information in the form of long nucleotide sequences. As a result of the work of the laboratory for research of biomechanical systems of the Mechanical Engineering Research Institute of the Russian Academy of Sciences in 2020, universal hyperbolic rules for the cooperative organization of genomes of higher and lower organisms were discovered $[4,5]$. These rules are associated with harmonic progression (1), which has long been known in various fields of science and has now received the status

Corresponding author: spetoukhov@gmail.com 
of an important participant in genetic informatics, capable of influencing the characteristics of inherited biosymmetries:

$$
1 / 1,1 / 2,1 / 3,1 / 4, \ldots, 1 / n
$$

In this article, we consider the mathematical connections of this progression with the groups of projective and Möbius transformations, on which nonEuclidean symmetries are based. Besides we consider fractal-like features of long nucleotide sequences in helical DNA, which are also related to the harmonic progression (1) and give new materials to the known hypothesis about DNA double helix as a helical antenna. The article contains 5 sections and conclusions.

\section{Computational approaches, models}

Our main computational approaches and models of biological structures are based on the mathematical properties of harmonic progression (1), which were studied by Pythagoras, Orem, Leibniz, Newton, Euler, Fourier, Dirichlet, Riemann and many other researchers in connection with various problems of physics and mathematics. Nowadays this list is supplemented with the tasks of studying its role in the genetic information fundamentals of living matter and the structuring of inherited biological systems. Its historically known name - "harmonic progression" - sequence (1) was received due to its connection with a number of harmonics in music (or a number of lengths of standing waves when vibrating a stretched string). This article presents the results of an analysis of the conjugation of this progression with the main invariant of projective geometry, called the double ratio (as well as the wurf and anharmonic ratio). In the one-dimensional case, the group of projective transformations and the group of Möbius transformations coincide, and the double relation is simultaneously an invariant of both projective and Möbius transformations. In our research on nonEuclidean biosymmetries, we actively use the provisions of the famous Erlangen program of F. Klein, which defined geometry as the science of invariants of the transformation group. In accordance with this program, each group of transformations has its own geometry, which has its own individual set of invariants. In particular, the groups of projective and Möbius transformations define projective and Möbius geometries (the latter is also called the geometry of reciprocal radii or conformal geometry).

The cross-ratio (or the double ratio or the wurf) of the four numbers a, b, c, d is determined by the expression (2):

$$
(a b, c d)=\{(\mathrm{c}-\mathrm{a})(\mathrm{d}-\mathrm{b})\} /\{(\mathrm{c}-\mathrm{b})(\mathrm{d}-\mathrm{a})\}
$$

If the four numbers $a, b, c, d$ correspond to the coordinates of four points on the number line, then expression (2) consists of the lengths of the corresponding segments between the indicated pairs of points. Under projective transformations of such a straight line, the lengths of the segments and the simple relations between them change, but the value of the cross-ratio remains unchanged.

\section{Some results: projective symmetries in the harmonic progression}

One of the properties of the harmonic progression (1) is that the value of the cross-ratio of any of its four neighboring numbers is equal to 4/3. Any four adjacent numbers in a series of natural numbers $1,2,3, \ldots, n$ also have the same value of the cross-ratio. This means that the harmonic progression (1) and the natural series of numbers are projectively equivalent to each other (Fig. $1)$.

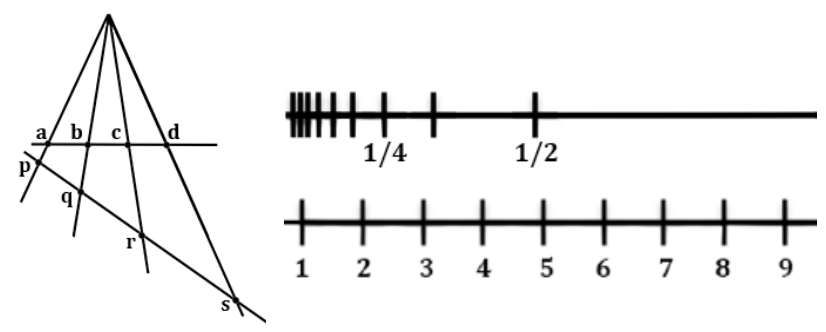

Fig. 1. Examples of projective transformation. Left: a central projective transformation changes the lengths of the line segments and their simple ratios, but retains the magnitude of the cross-ratio. Right: the location of the numbers of the harmonic progression (1) and a series of natural numbers on the number line.

The connection of the harmonic progression (1) with the cross-ratio leads to the following conclusions:

1. Various fragments of a harmonic progression can be considered as projectively symmetric to each other;

2. In the genomes of eukaryotes and prokaryotes, various hyperbolic sequences of oligomeric sums corresponding to the classes of A-, T-, C- and Goligomers [5] are projectively similar to each other with the high accuracy with which hyperbolic rules are fulfilled in them;

3. All investigated genomes of eukaryotes and prokaryotes with respect to their sequences of oligomeric sums are projectively similar to each other (with the high accuracy, with which hyperbolic rules are fulfilled in them).

Similar hyperbolic rules associated with harmonic progression (1) are fulfilled not only in genomic DNA sequences of nucleotides, but also in the studied long proteins (such as the Titin protein containing 33423 amino acids and presented at https://www.ncbi.nlm.nih.gov/protein/ ACN81321.1) in relation to oligomer sums in their 20 classes of amino acids. The point is that in the case of protein sequences of 20 types of amino acids, 20 equivalence classes can be analyzed, each of which is determined by the corresponding type of amino acid and combines all $n$ oligomers (fragments of length $n$ ) of a given protein that begin with a given amino acid. For example, the amino acid Ala defines a class of Ala-oligomers, which includes all $n$-oligomers starting with this amino acid: the subset of Ala-doublets contains all 20 doublets that 
start with Ala (AlaAla, AlaArg, AlaAsn, .... AlaCys); the subset of Ala-triplets contains all 400 triplets that start with Ala (AlaAlaAla, AlaAlaArg,...., AlaCysCys), and so on. The quantitative compositions of these subsets of $n$-oligomers of each of these amino acid classes for different $n=1,2,3, \ldots$ (here $n$ is much less than the protein length) are mutually related with high accuracy and obey hyperbolic rules associated with harmonic progression (1). Accordingly, taking into account the mentioned connection of this progression with the crossratio, we can talk about the projective-geometric (as well as the Möbius-geometric) similarity of the named quantitative characteristics of the structure of long proteins such as the Titin protein [5].

Taking into account that the numerical characteristics of genetic sequences are associated with the main invariant of projective geometry, then it can be expected that projective-geometric and Möbius-geometric symmetries will appear in genetically inherited physiological structures. This allows us to consider in a new light the existence of the non-Euclidean symmetries previously established in the works of our Mechanical Engineering Research Institute of RAS in a wide variety of morphogenetic structures and growth processes in living bodies [3].

For example, the cross-ratio was applied to the analysis of growth changes in the longitudinal proportions of three-membered kinematic blocks in the human body: three-phalangeal fingers, three-membered limbs (shoulder-forearm-hand and thigh-calf-foot) and the entire three-membered body (in anthropology, the body is subdivided into the upper, trunk and lower segments). The value of the cross-ratio of the lengths of the three members in all these trebled blocks, at least during the entire postnatal period, is grouped around the reference value of 1.3. This is all the more interesting because the growth of the human body is essentially non-linear (Fig. 2): for example, during a person's life from birth, the upper part of the body grows 2.4 times, the trunk - 2.8 times, and the lower part - 3.8 times. This invariance of the cross-ratio value turns out to be largely true not only for normally developed people, but also for dwarfs and giants. This is also true for a wide range of highly organized animals.

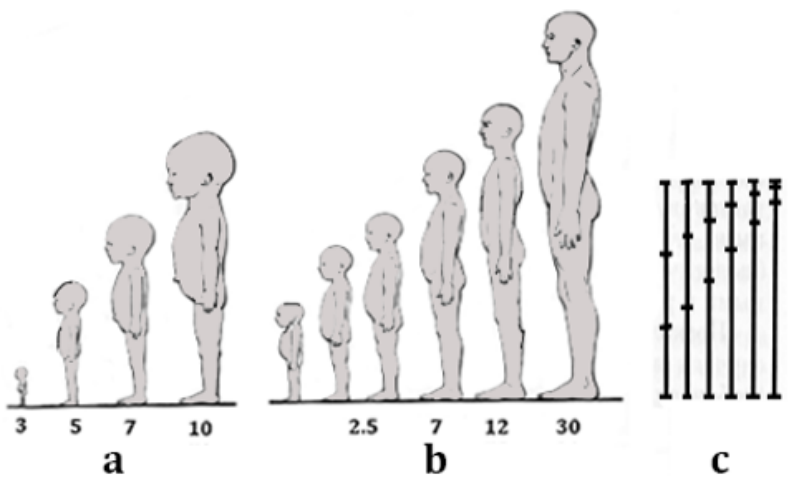

Fig. 2. Age-related changes in the proportions of the human body (from [3]). a, b - antenatal and postnatal stages: a - in the lunar months; b - in years (first newborn on the left); c examples of projectively equivalent three-term segments, the cross-ratio values of which are equal to 1.3.
We note additionally that projective-geometric transformations and the cross-ratio as their main invariant are mathematical tools, on which the study of visual perception and the creation of methods of computer vision, pattern recognition, and artificial intelligence are largely based. A huge number of publications are devoted to this topic (see, for example, $[6,7]$. The study of the relationship between the genetic system and genetically inherited biological structures, which are connected with non-Euclidean symmetries and harmonic progression, continues, including for the development of genetic biomechanics and algebraic biology. These issues, in particular, are considered at the International Interdisciplinary Seminar "Algebraic Biology and Systems Theory" (https://www.youtube.com/channel/UC8JLsuRzzPsRiH wrwEjMCtw ).

The creators of quantum mechanics P. Jordan and (later) E. Schrödinger pointed out the key difference between living bodies and inanimate ones: inanimate objects are controlled by the average random motion of their millions of particles and the motion of an individual particle is not essential for the whole; on the contrary, in a living organism, the chosen - genetic - molecules have a dictatorial (!) influence on the entire organism (see the history of quantum biology [8]). In accordance with this statement, in our research we focus on the properties of DNA information sequences and study their influence on inherited biological structures.

Jordan in his work on quantum biology claimed that life's missing laws were the rules of chance and probability of the quantum world [8]. From the standpoint of Jordan's statement, the study of probabilities or frequencies of n-plets in long DNA sequences is important for discovering hidden biological laws and for developing quantum biology. Our study is closely connected with this direction of thoughts and, in particular, gave the following model.

The alphabet of 4 nucleotides of DNA is endowed with binary opposition traits "purine-or-pyrimidine" and "strong-or- weak hydrogen bonds". Taking this binaryoppositional indicators into account, one of the coauthors of this article created a quantum-information model of long DNA-texts, where notions of quantum qubits and their tensor products are used $[4,5]$.

In this model, 2n-qubit representations of long DNA texts were obtained as quantum chains from the corresponding n-plets. This quantum information model makes it possible to predict many non-trivial numerical characteristics of long DNA texts, again associated with the harmonic progression $1,1 / 2,1 / 3,1 / 4,1 / 5, \ldots, 1 / n$. For example, the model predicts that in long DNA, the sums of n-ples starting with any of the 16 doublets are mutually related: the ratios of the sums of triplets, tetraplets, etc. to the sum of the selected doublet will be equal to the ratio of the second term $1 / 2$ of the harmonic progression (1) to its subsequent members $1 / 3,1 / 4,1 / 5$, ..., i.e. are equal to members of the following sequence:

$$
3 / 2,4 / 2,5 / 2,6 / 2,7 / 2, \ldots
$$


Similarly, in long DNA, the sums of $n$-ples beginning with any of 64 triplets are mutually related: the ratios of the sums of tetraplets, pentaplets and so on to the sum of the selected triplet are equal to the ratio of the third term of $1 / 3$ of the harmonic progression to its subsequent members $1 / 4,1 / 5,1 / 6, \ldots$ that is, equal to members of the the following sequence:

$$
4 / 3,5 / 3,6 / 3,7 / 3,8 / 3, \ldots
$$

These predictions of the quantum information model were confirmed with high accuracy under analyzing long DNA texts in genomes $[4,5]$.

One can note that the cross-ratio of any of four neighboring numbers in numeric sequences $(3,4)$ and similar them is again equal to $4 / 3$ as in the harmonic progression (1).

The effectiveness of the quantum-information model is evidenced by numerical data in favor of the fact that living organisms are quantum-information entities. This echoes the assumptions of many authors about the quantum-information basis of living things.

\section{Other results: DNA epi-chains and helical antennas}

Long DNA-texts have another previously unknown property associated with the harmonic progression and the so-called epi-chains of DNA. This property, which is described below, speaks of a fractal-like structure of these texts.

If in the DNA-texts of genomes we take only each $k$ th letter $(k=2,3, \ldots, 10, \ldots, 50, \ldots, 100, \ldots)$, then new shortened DNA-texts are formed with new sequences of letters A, T, C, G (Fig. 3). These shortened DNA-texts are called DNA epi-chains of corresponding order $k$ [9]. It turns out that - for each such DNA-text - the same hyperbolic rules, connected with the harmonic progression, are fulfilled with high precision (it is supposed that $k$ is much less than the length of the considered long DNA-text).
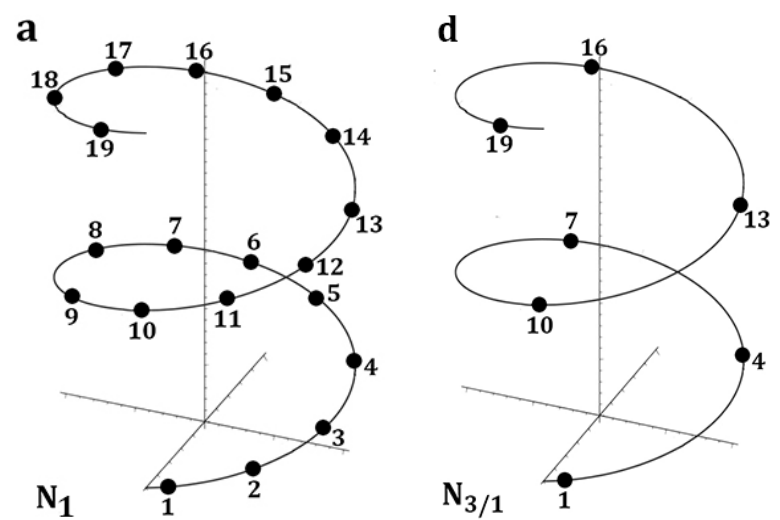

Fig. 3. Schematic representations of a single-stranded DNA and one od the examples of epi-chains of nucleotides; a, the sequence $\mathrm{N}_{1}$ of numerated nucleotides of the DNA strand; $\mathbf{d}$, the epi-chain of the third order $\mathrm{N}_{3 / 1}$, which contains nucleotides with numbers 1-4-7-10-... .
The harmonic progression since the time of Pythagoras is associated with harmonics of music and standing waves in resonators. This allows us to assume that the connection between the harmonic progression and the numerical fractal structure of helical DNA-texts is of a resonant nature and reflects the existence of a certain system of standing waves. This is consistent with the known hypotheses that helical DNA is the helical antenna of electromagnetic waves, and therefore, like other helical chiral biomolecules, emits and absorbs electromagnetic waves of a certain circular polarization, which provides biomolecules with the possibility of exchanging radio waves of selective polarization. Many types of organisms are endowed with polarizing vision.

Helical antennas, including fractal ones, are important in space communications, radars, and cellular telephony. They - unlike other antennas - generate circularly polarized radio waves. They are insensitive to manufacturing defects and disturbances in the relative orientation of the transmitting and receiving antennas. Chirality of bio-structures and circular polarization of their electromagnetic waves are important for pharmacology, physiotherapy, etc.

An analog of the helical antenna was recently discovered by Swedish scientists in the tail of the spermatozoid. It has been suggested that these spirals act as a CCTV camera for the spermatozoids to bypass obstacles and search for the egg. [10].

Antenna-like helical structures are involved in the biomechanics of coordinated movements. For example, the unicellular organism Mixotricha paradoxa moves due to the 250 thousand spiral bacteria Treponema spirochetes on its surface, whose helical flagella are been twisting cooperatively, providing purposeful movement.

Electrical and mechanical vibrations in living bodies are closely related, since many of their structures are piezoelectric: nucleic acids, actin, dentin, tendons, bones, etc. Thus, electromagnetic phenomena are accompanied in biological bodies by the phenomena of vibration mechanics.

\section{Some concluding remarks}

Gr. Mendel discovered in his experiments on the crossing of organisms that the inheritance of their traits occurs according to algebraic rules, despite the colossal complexity and heterogeneity of the molecular structure of living bodies. In this regard, he can be considered the founder of algebraic biology.

According to Mendel's law of independent inheritance of traits, information from the level of DNA molecules dictates the macrostructure of living bodies through many independent channels, despite strong noise and interference. For example, hair, eye, and skin colors are inherited independently of each other. This determinism is provided by unknown algorithms of multichannel noise-immune coding. Accordingly, each organism is a multichannel noise-immune coding machine (in which DNA plays a dictatorial role).

Our studies of nucleotide DNA sequences in the genomes of higher and lower organisms reveal the 
existence in them of a system of quantitative hyperbolic rules of a universal nature, which are related to the harmonic progression (1).

The mathematical properties of this historically known progression, used in various fields of science and now found in the quantitative characteristics of molecular genetic informatics, indicate that the algebraic structures of genetic informatics can be a basis of nonEuclidean symmetries in the inherited morphology and biomechanics of movements [11]. On this path, new classes of models in biomechanics, psychophysics of sensory perception and algebraic biology, as well as new approaches in biotechnology and biotechnical systems, including artificial intelligence systems, are being developed.

The discovery of the relationship between genomes and harmonic progression also raises important questions about the relationship between the genetic harmony of organisms and musical harmony. These issues are now being dealt with by the special "Center for Interdisciplinary Research of Musical Creativity" of the Moscow State Tchaikovsky Conservatory.

Additional materials were obtained to the wellknown statement based on the achievements of molecular biology: life is a partnership between genes and mathematics [12].

\section{Conclusions}

The present work leads us to the following conclusions :

1. The harmonic progression (1) is connected with the cross-ratio, which is the main invariant of projective geometry. This fact gives pieces of evidence that long DNA nucleotides sequences, whose structures are related to the harmonic progression, consist of fragments which are projective symmetric to each other. It leads to a new - genetic - consideration of the known phenomena of non-Euclidean symmetries in genetically inherited biological structures ;

2. The fractal-like structure of long DNA nucleotide sequences and their epi-chains of different orders is connected with the harmonic progression and the crossratio and projective symmetries;

3. The received results give new materials to a consideration of DNA double helix as a helical antenna, which emits and absorbs electromagnetic waves of circular polarization.

The authors are grateful to G. Darvas, E. Fimmel, I. Stepanyan, and G. Tolokonnikov for assistance in the work and discussion of the results.

\section{References}

1. H. Weyl, Symmetry (Princeton University Press., 2016)

ISBN-13:978-0691173252, ISBN-10:0691173257

2. A.V. Shubnikov, V.A. Koptsik, Symmetry in Science and Art, 3rd ed. (Moscow: RChD, 2004).
3. S.V. Petoukhov, Computers \& Mathematics with Applications, 17(4-6), 505-534 (1989)

4. S.V. Petoukhov, Biosystems, 198, 104273 (2020)

5. S.V. Petoukhov, Preprints 2020, 2020050471 (2020) DOI:10.20944/preprints202005.0471.v2.

6. R. Gonzalez, R. Woods, Digital Image Processing (Prentice Hall, 3rd ed, 2007)

7. D.A. Forsyth, J. Jean Ponce, Computer Vision: A modern Approach (Pearson, 2nd ed, 2011)

8. J. McFadden, J. Al-Khalili, The origins of quantum biology. Proceedings of the Royal Society A, 474(2220), 1-13 (2018)

9. S.V. Petoukhov, Preprints 2019, 2019040011 (2019), DOI:10.20944/preprints201904.0011.v2.

10. D. Zabeo, J.M. Heumann, C.L. Schwartz, A. Suzuki-Shinjo, G. Morgan, P.O. Widlund, J.L. Höög, Sci.Reports, 8(1), 2727 (2018)

11. N.A. Bernstein, Essays on the physiology of movements and the physiology of activity (Moscow, Medicine Publ., 1966).

12. I. Stewart, Life's Other Secret: The New Mathematics of the Living World (New York, Wiley, 1999). 